\title{
Abordagem fisioterapêutica em um paciente com neuromielite óptica: relato de caso
}

\section{Physyotherapeutic approach in a patient with neuromyelitis optic: case report}

Raquel de Oliveira Garcia ${ }^{1}$

Hassan Hussein Hamdan²

Cristiane Fernanda Gessinger ${ }^{3}$

\section{RESUMO}

A Neuromielite óptica, também chamada Doença de Devic, é uma doença idiopática, grave e rara. É caracterizada por ser inflamatória, e leva à desmielinização, principalmente do nervo óptico (neurite óptica) e da medula espinhal (mielite transversa). A literatura é escassa na descrição do tratamento fisioterapêutico destes casos. Objetivo: Descrever a abordagem fisioterapêutica em pacientes com Neuromielite Óptica. Descrição: Paciente de 59 anos, branca e do sexo feminino. Sintomas iniciaram em 2003, porém o diagnóstico de Neuromielite Óptica foi concluido em 2008. Iniciou o tratamento fisioterapêutico em 2008 e vem sendo atendida três vezes por semana com cinesioterapia e terapia manual.

PALAVRAS-CHAVE:

Fisioterapia; Doença de Devic; Neuromielíte Óptica; Abordagem fisioterapêutica.

\footnotetext{
${ }^{1}$ Acadêmica do curso de Fisioterapia do Centro Universitário Metodista IPA, Porto Alegre-Rio Grande do Sul- Brasil.

${ }^{2}$ Acadêmico do curso de Fisioterapia do Centro Universitário Metodista IPA, Porto Alegre-Rio Grande do Sul- Brasil.

${ }^{3}$ Fisioterapeuta, Ms. Docente e Supervisora de estágio do curso de Fisioterapia do Centro Universitário Metodista IPA, Porto Alegre-Rio Grande do Sul- Brasil.
} 


\section{ABSTRACT}

The neuromyelitis optics, also known as Devic's disease is an idiopathic disease, severe and rare. It is characterized by inflammation, leading to demyelination, particularly the optic nerve (optic neuritis) and spinal cord (transverse myelitis). The literature is scarce in the description of the physical therapy treatment of these cases. Objective: To describe the physical therapy approach in a patient with neuromyelitis optics.Description: Patient 59, white and female. Symptoms started in 2003, but the diagnosis of neuromyelitis optics was completed in 2008. She began physical therapy in 2008 and has been served three times a week with kinesiotherapy and manual therapy.

\section{KEYWORDS:}

Physiotherapy; Devic's disease; Neuromyelitis optics; Physical therapy approach. 


\section{Introdução}

A Neuromielite óptica (NMO) encontra-se na classe das doenças desmielinizantes do sistema nervoso central (SNC). Esta doença pode apresentar-se de diversas formas: a mielite e a neurite, que podem ocorrer de forma simultânea ou com um intervalo de tempo entre elas; além disso, a doença pode ter um curso monofásico, sem novos eventos, ou recidivante, com crises separadas por meses ou até anos. Uma vez que existe envolvimento da medula espinhal geralmente sob forma de mielite transversa, o quadro clínico é de uma paraparesia, com perda sensorial bilateral e disfunção esfincteriana. O comprometimento do nervo óptico ocasiona amaurose que pode evoluir para cegueira, geralmente no caso de doença monofásica, porém o curso da doença é imprevisível. $1,2,3,4,5,6$.

Na maioria dos casos, uma etiologia específica não é identificada, mas um mecanismo imunológico de dano tecidual parece provável ${ }^{2}$. A neurite óptica na NMO, geralmente é mais grave e de recuperação incompleta. As crises, habitualmente são de intensidade moderada a severa e as remissões geralmente incompletas ${ }^{3}$.

Mais da metade dos doentes apresenta amaurose total de pelo menos um olho e/ou incapacidade para deambular de forma autônoma ao fim de cinco $a_{n}{ }^{3}$. A NMO é rara na população caucasiana, representando menos de $1 \%$ das doenças desmielinizantes do SNC, apresenta alta incidência na população Asiática, Africana e Sul-Americana. É três vezes mais frequente no sexo feminino (na forma recidivante), com início, em média, aos 40 anos de idade 4 .

Recentes avanços têm demonstrado que na maioria dos casos a NMO é recorrente e resulta de alterações inflamatórias por ataque à aquaporina-4, uma proteína localizada nos pés dos astrócitos na barreira hematoencefálica ${ }^{5}$. Em 2005 identificou-se um biomarcador específico para NMO (73\% de sensibilidade e $91 \%$ de especificidade), ou autoanticorpo NMO-IgG, um elemento crucial na individualização desta síndrome ${ }^{3,7}$.

O objetivo deste estudo foi descrever a abordagem fisioterapêutica com uma paciente que apresenta esta patologia, já que são escassas as publicações sobre a fisioterapia nestes casos. A abordagem fisioterapêutica para essa paciente teve como objetivos realizar um trabalho global e funcional, a fim de promover maior qualidade de vida e independência para a paciente, através do fortalecimento da musculatura de membros superiores e inferiores, com uso de exercícios de cadeia cinética fechada e diagonais de FNP (Facilitação Neuromuscular Proprioceptiva), assim como a intervenção na reeducação postural, melhora do equilíbrio, coordenação motora e propriocepção.

\section{Relato do Caso}

A paciente relatou ter início dos sintomas em 2003, quando sentiu sua visão turva e sensações de fraqueza durante o dia, então neste mesmo ano realizou o exame de ressonância nuclear magnética, onde apresentou uma pequena inflamação no nervo óptico direito, porém com laudo não conclusivo para alguma patologia.

Os sintomas tornaram-se mais recorrentes e em 2006 a ressonância indicava sinais sugestivos de neurite óptica à esquerda. Em 2007 realizou nova ressonância com aspectos sugerindo processo inflamatório (neurite) à direita e área de desmielinização, ou mesmo pequena área de dano vascular à esquerda; lesão medular no nível de C2 ( $2^{\mathrm{a}}$ vértebra cervical) até $\mathrm{C} 5$ ( $5^{\mathrm{a}}$ vértebra cervical) e T1 ( $1^{\mathrm{a}}$ vértebra torácica) até T4 (4 vértebra torácica), sugestivo de patologia desmielinizante.

Em 2008 realizou o exame específico de NMO-IgG, onde foram encontradas alterações nos anticorpos aquaporina 4, sendo indicativo de Neuromielite Óptica. A ressonância nuclear magnética indicou comprometimento de pedúnculos cerebelares superiores.

De 2009 a 2012 apresentou ressonâncias semeIhantes às já descritas. Em 2014 a ressonância nuclear magnética indicou comprometimento em bulbo à direita.

Desde o fechamento do diagnóstico a paciente foi encaminhada para a fisioterapia em um ambulatório de fisioterapia de um hospital de Porto Alegre, onde recebe atendimento até o presente momento. $\mathrm{O}$ atendimento é realizado por estagiários do curso de fisioterapia com supervisão. 
Quando às crises, identificadas pela própria paciente pelo agravamento dos sintomas, duram mais que 24 horas, é necessária a internação para o tratamento de Pulsoterapia com Corticosteróide venoso em altas doses e plasmafereses, usadas no tratamento das fases agudas, enquanto imunossupressores devem ser usados na profilaxia das recorrências $^{7}$. A necessidade da hospitalização durante a pulsoterapia ocorre devido aos possíveis efeitos colaterais que o paciente pode apresentar durante a infusão da droga ${ }^{8}$.

\section{Discussão}

Atualmente a paciente encontra-se com diagnóstico fisioterapêutico de fraqueza muscular de todos os grupos musculares de membros superiores e inferiores, sendo classificada como grau 4 nos testes de função muscular descritos segundo Kendall ${ }^{18 .}$ Apresenta restrição e dor ao movimento de rotação cervical ativa e inclinações devido a presença de contraturas em trapézio superior esquerdo. Apresenta teste de equilíbrio em apoio unipodal positivo em membro inferior esquerdo. Diminuição de amplitude de movimento para rotação e inclinação cervical e para os movimentos de flexão e extensão do ombro, ambos do lado esquerdo.

Foram utilizadas como condutas fisioterapêuticas:

Intervenção postural com enfoque na cintura escapular onde deve-se realizar a depressão da escápula e crescimento axial.

Na posição em pé ou sentada, a gravidade imprime sobre o corpo uma força que achata e aumenta as lordoses. Na execução dos exercícios, antes da preocupação com a diminuição das curvas da coluna, o eixo vertebral deve crescer para exercer força contrária à da gravidade. Na reeducação do movimento é importante garantir o espaço entre uma vértebra e outra, onde durante o processo inspiratório, a cabeça e a cintura pélvica são tracionadas em direções opostas, a cabeça para cima, e a cintura pélvica para baixo. Esse crescimento é mantido durante a expiração9.

Esta intervenção se dá a partir do conhecimento de que o indivíduo estando na posição em pé ou sentado, a gravidade aplica sobre o corpo uma força que diminui as suas lordoses fisiológicas. Então, durante os exercícios, o eixo vertebral deve crescer, exercendo uma força contrária à da gravidade. Para garantir espaço entre uma vértebra e a outra. Durante o processo inspiratório realiza-se uma força de tração, onde a cabeça e cintura pélvica se movem em direções opostas, este crescimento deve ser mantido durante todo o ciclo respiratório9.

Exercícios de propriocepção e equilíbrio, com uso da prancha proprioceptiva.

O equilíbrio é um processo complexo que depende da integração da visão, da sensação vestibular e periférica, dos comandos centrais e respostas neuromusculares e, particularmente, da força muscular e do tempo de reação. Para obter um melhor equilíbrio, um indivíduo procura manter o seu centro de massa corporal dentro dos seus limites de estabilidade, sendo determinada pela habilidade em controlar a postura sem alterar a base de suporte ${ }^{10}$. A propriocepção é definida como a percepção consciente e inconsciente da posição do membro no espaço, músculos (proprioceptores periféricos) e dos sistemas vestibular e visual ${ }^{11}$.

O equilíbrio depende da integração de diversos fatores: visão, sistema vestibular, informações periféricas, comandos centrais, respostas neuromusculares, força muscular e tempo de reação. Para manter-se em equilíbrio, o indivíduo procura manter seu centro de massa corporal dentro dos limites da sua estabilidade. A propriocepção é definida como a percepção consciente e inconsciente da posição do membro no espaço, onde receptores periféricos (proprioceptores) enviam estas informações para o SNC integrá-la ${ }^{10,11}$.

Liberação miofascial em trapézio superior esquerdo.

Técnica que atua com mobilizações manuais da fáscia, que são executadas para aumentar a amplitude de movimento, aliviar a dor e restaurar a quantidade e qualidade normal dos movimentos ${ }^{12,19}$.

Técnicas manuais, onde a partir da liberação da fáscia, se busca o aumento da amplitude de movimento, alívio da dor e restauração da qualidade cinéticofuncional. 
Fortalecimento muscular de membros superiores e inferiores com uso das diagonais do FNP D2 flexora e extensora.

A facilitação neuromuscular proprioceptiva (FNP) fornece ao terapeuta ferramentas necessárias para ajudar o paciente a atingir uma função motora eficiente. A FNP tem o caráter, como sugere o próprio nome, tornar fácil o movimento, envolvendo nervos e músculos, trabalhando-se os receptores sensoriais que enviam informações relacionadas ao movimento e posicionamento corporal. A FNP tem o objetivo primário de facilitar o paciente a alcançar o mais alto nível funcional reforçando e utilizando o que ele pode fazer. O aprendizado motor é o processo pelo qual o sistema nervoso modifica suas respostas motoras. Sendo um processo implícito, tem como principais características o desenvolvimento lento, baseado em um grande número de repetições, cujos resultados só podem ser verificados através da modificação no desempenho $10,13,14,16,17,22,23$.

Agachamento em cadeia cinética fechada.

Realiza-se visando o fortalecimento da musculatura extensora de quadril e extensora de joelho. 0 glúteo máximo geralmente é ativo apenas quando o quadril está em flexão, como durante o ato de subir escadas ou andar de bicicleta, ou quando a extensão do quadril for resistida. A cadeia cinética inferior é um sistema especializado projetado para a locomoção humana, dor, fraqueza e doença, podem ocasionar distúrbios no ritmo normal da marcha ${ }^{19}$. O exercício é realizado com o intuito de obter e manter funcionalidade para gestos fundamentais como o subir e descer escadas, elevar-se de uma cadeira e troca de postura de sentado para em pé.

\section{Exercício de ponte.}

Visando fortalecer a musculatura glútea, isquiotibiais e quadríceps. A ponte utiliza o peso do corpo como resistência dos extensores e abdutores do quadril $^{19}$. Para a marcha, estão ativos os grupos musculares que equilibram a pelve e suportam o tronco (glúteo médio, glúteo mínimo, glúteo máximo, eretor da coluna e tensor da fáscia lata). Eles contraem excentricamente, alongando-se e permitindo a pelve cair $5^{\circ}$ para baixo. É a contração do glúteo máximo nesse período de impato do calcâneo que evita a queda da pelve para a frente, no sentido da locomoção. Os abdutores do quadril (glúteos médio e mínimo) predominam no início da fase de apoio, enquanto os adutores (adutor longo, adutor curto, adutor magno, pectíneo e grácil) são mais atuantes no final da fase de apoio e início da oscilação ${ }^{24}$. Sendo músculos utilizados na marcha, nas trocas de postura de sentado para de pé este trabalho contribui para a manutenção da funcionalidade à medida que são reforçados

Alongamentos ativos estáticos com duração de 30 segundos.

O alongamento é um termo geral usado para descrever qualquer manobra terapêutica elaborada para aumentar o comprimento de estruturas de tecidos moles patologicamente encurtados e deste modo aumenta a amplitude de movimento. Realizamos um alongamento estático quando tecidos moles são alongados um pouco além do ponto de resistência do tecido, e então mantidos na posição alongada por um longo tempo com uma força de alongamento.A diminuição da função muscular resulta de uma variedade de causas, incluindo o processo patológico, desuso, ou espasticidade dominante sobre os músculos antagonistas. Para aumentar ou manter a força, exercícios resistidos progressivos e programas de flexibilidade devem ser realizados diariamente. O alongamento atua diretamente e indiretamente na elasticidade e integridade muscular participando da função de proporcionar agilidade de movimento. $\mathrm{O}$ procedimento de elasticidade músculo tendinosa propicia ajustamentos posturais importantes para o processo de recuperação ${ }^{15,19,20,21,23,25}$.

\section{CONSIDERAÇÕES FINAIS}

A abordagem fisioterapêutica, apesar de pouco descrita na literatura para esses casos, visa melhorar a capacidade funcional e a capacidade de realização das atividades de vida diária, além de prevenir complicações, motoras e ventilatórias e proporcionar maior independência ao paciente.

É importante lembrar que as condutas são constantemente modificadas para que a paciente se sinta motivada, tendo sempre novos desafios, já que o 
tratamento teve início há seis anos, o que é um tempo bastante prolongado. A paciente refere que o tratamento se torna mais dinâmico, sendo um ponto positivo. Também, que devido à fisioterapia tem mantido a funcionalidade, mesmo com o prognóstico desfavorável previsto pela literatura.

O tratamento em grupo seria uma excelente opção para a paciente, porém por serem raros os casos, ainda não foi possível constituir um grupo.

\section{REFERÊNCIAS}

MARRA, E.S.; FOSS, M.H.D. Intervenção Fisioterapêutica na Doença de Devic: um estudo de caso. Arquivos de Ciências da Saúde. Vo.17, supl.1,2010.

SILVA, A.R.; BARROS, V.T.S.; ROTTA, N.T.; OHLWEILER, L.; STONE, I.; MELLO, L.R. Doença de Devic: relato de caso. Jornal de Pediatria. Vol.77. Nº.2001.

PEIXOTO, I.; ERMIDA, V.; TORRES, A.; APARÍCIO, M.I.; ANDRÉ, R.; CALDAS, J. Doença de Devic. Acta Med Port.2010;23:263-266.

SOUZA, C.S.; BROOKS, J.B.B.; OLIVEIRA, C.L.S.; FRAGOSO, Y.D. Neuromyelitisoptica with very late onset. Arquivo de neuropsiquiatria. 71(8). 556-557,2013.

FERREIRA, M.L.B. Comparing neuromyelitisoptica and multiple sclerosis severity: is there a difference? Arquivo de Neuropsiquiatria. 2013; 71(5): 271-272.

PEIXOTO, M.A.L. Devic'sneuromyelitisoptica. Arquivo de neuropsiquiatria, 2008;66(1): 120-138.

SATO, D.; CALLEGARO, D.; PEIXOTO, M.A.L.; FUJIHARO, K. On behalf Brazilian Committee for treatment and research in multiple sclerosis(BCTRIMS). Treatment of neuromyelitisoptica: an evidence based review. Arquivo Neuropsiquiatria. 2012; 70(1): 59-66.

ROZENCWAJG, D.; NUNES, C.F.P.; SAKUMA, L.M.; LASELVA, C.R.; ROZA, B.A. Assistência de enfermagem ao paciente com corticosteroide. Artigo de revisão. Einstein. 2008; 6(4): 491-6.

BERTAZZO, I. Reeducação do movimento. Cérebro ativo, corpo vivo. São Paulo. Manole. 2012.

DOS SANTOS, T.; PERACINI, T.; FRANCO, P.; NOGUEIRA, R. Facilitação neuromuscular proprioceptiva na doença de $\mathrm{Pa}$ rkinson: relato da eficácia terapêutica. Fisioterapia e Movimento. Curitiba. V.25.N². P.281-289. Abr/Jun.2012
CAMARGOS, F.; LANA, D.; DIAS, R.; DIAS, J. Estudo da propriocepção e desempenho funcional em idosos com osteoartrite de joelhos. Rev Brasileira de Fisioterapia. Vol.8. N¹.2004.

ARRUDA, G.; STELLBRINK, G.; OLIVEIRA, A. Efeitos da liberação miofascial e idade sobre a flexibilidade de homens. Terapia Manual. 2010.

ALENCAR, R.; CORDEIRO, T.A.P.; CAVALCANTI, P. Facilitação Neuromuscular proprioceptiva em tatame na reaquisição de funções na lesão medular. Revista Neurociências. 2011. JUNIOR, C.; DALL'AGILO, M.; GONÇALVES, C.; MARTINS, M.; MAIA, T. Facilitação neuromuscular proprioceptiva na esclerose lateral amiotrófica. Revista Fisioterapia S Fun. Fortaleza.

KISNER, C.; COLBY, L. Exercícios terapêuticos: fundamentação e técnicas. São Paulo. Manole. 5 ed.2009.

VOSS, D. Facilitação neuromuscular proprioceptiva. São Paulo. Panamericana. $3^{\circ}$ ed.1987.

REICHEL, H. Método Kabat. Facilitação neuromuscular proprioceptiva. São Paulo. Premier.1998.

KENDALL, F. P.; MCCREAY, E. K., PROVANCE, P. G. Músculos: provas e funções, $5^{a}$ edição. São Paulo: Manole, 2007.

DUTTON, Mark. Fisioterapia ortopédica: exame, avaliação e intervenção. $2^{\circ}$ edição- Porto Alegre: Artmed, 2010.

UMPHRED, Darcy Ann. Fisioterapia Neurológica. $2^{\circ}$ ed. São Paulo: Manole, 1994.

FIDELIS, L. A. S. Fisioterapia em neurologia: uma ideia cognitiva. São Paulo: Robe, 2001.

ADLER, S. S.; BECKERS, D. \& BUCK, M. P.N.F. Facilitação Neuromuscular Proprioceptiva. $2^{\circ}$ Edição, Editora Manole, 2007.

NUNES, L.E.; MARTINS, R.A.S.; MACEDO, A.B. A eficácia da associação das técnicas de alongamento, facilitação neuromuscular proprioceptiva e controle postural em adolescente com hemiparesia- estudo de caso. Revista eletrônica Saúde CESUC, 2010.

OSVANDRÉ, L.; BARROS, Filho. Exame físico em Ortopedia $2^{\circ}$ edição. São Paulo: Sarvier, 2001.

BATTISTI, A.; SILVA, D.O.; KESSLER, E.C.H.; ALBERT, J.; KIARA, P.Y.; VILAGRA, J.M.; BERTOLINI, C.R.F. Comparação do alongamento estático de 15 ou 30 segundos na extensibilidade de isquibiatibiais. ConScientiae Saúde, 2012, 11(4): 566-572. 